\title{
Verhaltensmedizin in der Neurologie: State of the Art
}

\author{
Bernd Leplow \\ Institut für Psychologie, Martin-Luther-Universität Halle-Wittenberg, Halle/Saale, Deutschland
}

\section{Schlüsselwörter \\ Verhaltensmedizin - Gelernter Nichtgebrauch . \\ Schlaganfall · Amyotrophe Lateralsklerose · \\ Brain-Computer-Interface · Epilepsie · Multiple Sklerose · Parkinson}

\section{Zusammenfassung}

Verhaltensmedizinische Interventionen setzen bei neurologischen Erkrankungen mit dem Ziel an, die gesellschaftliche Teilhabe so weit wie möglich zu erhalten oder wiederherzustellen. Dies kann über restitutive und kompensatorische Zugangswege mit oder ohne direkte Intervention am somatischen Substrat erfolgen. So lassen sich schlaganfallbedingte Paresen zumindest teilweise auch als Verlust des motorischen Gedächtnisses erklären. Durch lernpsychologisch begründete Interventionen ist neben der funktionellen Verbesserung auch eine Wiederherstellung der somatosensorischen Repräsentation möglich. Über EEG-Feedback-gestützte Brain-Computer-Interfaces kann dagegen die bei nahezu kompletten Lähmungen der Willkürmotorik ausgefallene Kommunikation mit der Umwelt kompensiert werden. Therapeutische Maßnahmen, die nicht direkt am somatischen Substrat ansetzen, zielen entweder auf die Wechselwirkung zwischen kurzfristigen, symptomatischen Verschlechterungen und situativen Auslösern oder auf den emotionalen und behavioralen Umgang mit einer somatischen Erkrankung. So ist eine situationsspezifische Symptomkontrolle unter anderem bei Anfallserkrankungen mit oder ohne NeurofeedbackUnterstützung möglich. Darüber hinaus kann die Behandlung psychischer Beschwerden und Störungen heute bei fast allen neurologischen Erkrankungen indiziert sein. Deshalb sollen mit diesem Beitrag die vielfältigen Möglichkeiten herausgestellt werden, die bereits jetzt zur verhaltensmedizinischen Unterstützung der Selbstregulation bei ganz unterschiedlichen neurologischen Krankheitsbildern zur Verfügung stehen.

\section{KARGER \\ Fax +497614520714 \\ Information@Karger.com}

www.karger.com

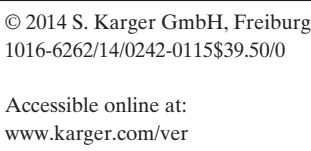

\author{
Keywords \\ Behavioral Medicine - Learned non-use . \\ Stroke - Amyotrophic lateral sclerosis . \\ Brain-computer interface - Epileptic seizures . \\ Multiple sclerosis · Parkinson
}

\section{Summary \\ Behavioral Medicine in Neurology: State of the Art} Numerous behavioral medicine interventions have been established to enhance participation with a large variety of neurological diseases. Compensation or restitution can be achieved using techniques directly addressed to CNS functions combined with behavioral methods or by means of behavioral methods alone. Based on the 'learned non-use' paradigm, restitution in hemiparesis is possible using 'Constraint-Induced Movement Training' and related techniques. With respect to conditions with near-to-complete loss of motor functions, compensation of speech production can be achieved using EEG feedback paradigms (brain-computer interfaces). Moreover, the interaction of neurological symptom exacerbation and specific cues can be modified by behavioral interventions with or without additional EEG feedback (e.g., seizure control). In addition, numerous approaches addressing emotional and behavioral factors associated with neurological diseases have been developed (in epilepsy, multiple sclerosis, Parkinson's disease, dementia, etc.). Focusing on the enhancement of self-regulation, basic principles of behavioral interventions are outlined for a variety of different neurological diseases. 


\section{Einführung}

Neurologische Krankheitsbilder weisen neben den motorischen und sensorischen Funktionseinbußen in der Regel eine Vielzahl emotionaler, behavioraler und kognitiver Veränderungen auf, die das alltägliche Leben und die zwischenmenschlichen Kontakte erheblich beeinträchtigen. Oft lassen sich jedoch keine klaren Zusammenhänge zwischen dem Schweregrad einer neurologischen Erkrankung und der erlebten Beeinträchtigung finden. So können sowohl geringgradige Ausprägungen mit einem hohen Leidensdruck als auch umgekehrt schwerste Verläufe mit einem erstaunlichen Ausmaß an Lebenszufriedenheit einhergehen. An dieser Diskrepanz von Funktionsbeeinträchtigung (nach der ICF-Terminologie: «Aktivitätseinschränkungen», früher: «disability») und mangelnder sozialer Teilhabe (Einschränkungen der «Partizipation», früher «handicap») setzen verhaltensmedizinische Interventionen mit dem Ziel an, die Teilhabe am gesellschaftlichen Umfeld trotz gegebener somatischer Beeinträchtigung soweit wie möglich zu erhalten oder wiederherzustellen. In einigen Fällen kann die körperliche Funktionseinschränkung auch direkt beeinflusst werden.

\section{Verhaltensmedizinische Zugänge in der Neurologie - eine Systematik}

Verhaltensmedizinische Interventionen haben ihren Ansatzpunkt entweder primär direkt am somatischen Substrat oder vorrangig an der Wechselwirkung von physiologischer Aktivität mit situativen, emotionalen und behavioralen Faktoren.

Der Ansatz des «gelernten Nichtgebrauchs» (learned nonuse) stellt ein Beispiel für einen direkten, über das somatische Substrat erfolgenden restitutiven Zugang dar (Tab. 1). Über ein psychologisch begründetes Training wird bei Schlaganfallpatienten die cerebrale Repräsentation des paretischen Bewegungsorgans beeinflusst und damit die krankheitsbedingte Funktionseinschränkung dauerhaft gebessert. Im Unterschied zur Physiotherapie handelt es sich hier aber nicht um eine reine Übungsbehandlung, sondern um einen verhaltensmedizinischen Ansatz, bei dem bereits die Ätiologie psychologisch begründet ist und die Intervention auf lernpsychologischen Gesetzmäßigkeiten aufbaut. Die Wiederherstellung erfolgt demnach sowohl über ein psychologisch aufgebautes Training als auch mithilfe der Psychoedukation und des systematischen Einsatzes von Belohnungen.

Mit einem Brain-Computer-Interface (BCI) wird der somatische Funktionsausfall dagegen nicht restituiert, sondern über die Nutzung noch intakter Funktionen des zentralen Nervensystems (ZNS) kompensiert (Tab. 1). Diese Technik beruht auf einem EEG-Feedback-Training, mit dem die willentliche Kontrolle von Hirnpotenzialen erlernt werden kann. Auf diese Weise ist es möglich, über die Änderungen dieser elektrischen Potenziale einen Cursor zu kontrollieren und damit auf einem Computerbildschirm Buchstaben, Wörter und Satzteile sowie Bedienungselemente anzusteuern. Auf diesem Weg wird es den Patienten mit nahezu komplettem Ausfall der Willkürmotorik ermöglicht, wieder mit ihrer Umwelt zu kommunizieren und ihr Erleben darzustellen - mit oft überraschenden Einblicken für Angehörige und Betreuer.

Neben diesen, über die direkte Beeinflussung des somatischen Systems laufenden restitutiven und kompensatorischen Interventionsansätzen erfolgt eine zweite grundlegende Behandlungsstrategie über die Beeinflussung der Person-UmweltInteraktion (Tab. 1). Hierfür gibt es wiederum 2 Zugangswege: zum einen primär über die situationsspezifische Symptomkontrolle und zum anderen über die direkte Beeinflussung des Krankheitsverhaltens und der Emotionalität. Die Wirkrichtung beider Ansätze ist also gegenläufig: Im ersten Fall wird zunächst die Symptomkontrolle verbessert und als Folge die Besserung des Befindens erwartet. Im zweiten Fall wird direkt die emotionale Seite des Krankheitsgeschehens beeinflusst und als Folge von einem geringeren Maß situationsspezifischer Exazerbationen ausgegangen. Selbstverständlich lassen sich beide Ansätze in der Praxis miteinander verbinden.

Die Verbesserung der situationsspezifischen Symptomkontrolle kann auf 2 verschiedenen Wegen erfolgen: entweder

Tab. 1. Unterstützung der Selbstregulation neurologischer Patienten

\begin{tabular}{|c|c|c|c|}
\hline \multicolumn{2}{|c|}{$\begin{array}{l}\text { Regulation physiologischer Signale: Intervention vorrangig über das } \\
\text { somatische Substrat }\end{array}$} & \multicolumn{2}{|c|}{$\begin{array}{l}\text { Verhaltensregulation: Intervention vorrangig über die Person-Kontext- } \\
\text { Interaktion }\end{array}$} \\
\hline Restitution des somatischen & Kompensation am somatischen & situationsspezifische & Krankheitsverhalten und \\
\hline Systems & System & Symptomkontrolle & Emotionalität \\
\hline \multicolumn{4}{|l|}{ Beispiele } \\
\hline «Learned Non-Use» bei & Brain-Computer-Interface bei der & Anfallskontrolle bei Epilepsien und & emotions- und \\
\hline Schlaganfallpatienten & Amyotrophen Lateralsklerose (ALS) & $\begin{array}{l}\text { Tics mit oder ohne Neurofeedback; } \\
\text { Kontrolle situationsspezifischer } \\
\text { Exazerbationen bei } \\
\text { Basalganglienerkrankungen }\end{array}$ & $\begin{array}{l}\text { problemfokussiertes Coping } \\
\text { bei allen chronischen } \\
\text { neurologischen Erkrankungen; } \\
\text { psychologische Anpassung an } \\
\text { die Folgen der Tiefen } \\
\text { Hirnstimulation }\end{array}$ \\
\hline
\end{tabular}


direkt durch die Steuerung cerebraler Funktionen oder ausschließlich über die Verhaltensebene. Beim direkten Zugang wird mithilfe von Neurofeedback-Verfahren die elektrische Hirnaktivität so konditioniert, dass die Frühphase eines epileptischen Anfalls rechtzeitig erkannt und dieser durch gelernte Veränderungen der Potenziale abgefangen werden kann. Auf diese Weise dienen EEG-Feedback-Verfahren dazu, anfallskorrelierte kortikale Aktivierungsmuster unter Kontrolle zu bringen und so die Anfallsfrequenz und -schwere zu begrenzen.

Ohne die Neurofeedback-Technologie ist dieses Ziel auf der Basis der «Habit Reversal»-Technik mit rein behavioralen Methoden (counter measures) erreichbar. Nicht nur psychomotorische Anfälle, sondern viele andere Symptome neurologischer Erkrankungen treten in unterschiedlichsten emotionalen oder physikalischen Auslösesituationen verstärkt auf. Das ist besonders in sozialen Situationen der Fall, in denen die emotionale Beteiligung eine weitere Verstärkung der Symptomatik nach sich zieht. Beispielhaft kann das bei den Erkrankungen der Basalganglien, also den Parkinson-, Dystonie- und Tic-Erkrankungen beobachtet werden. Hier wie auch bei den psychomotorischen Anfällen kommt es darauf an, die persönlichen Hochrisikosituationen ebenso zu erkennen wie die sich aufbauenden körperlichen Veränderungen. Gelingt dies rechtzeitig, kann mit spezifischen kompensatorischen Verhaltensmanövern wie Ablenkung, Konzentration, Entspannung oder anderen mentalen Strategien gegengesteuert werden, um so ein Überschießen des somatischen Geschehens abzufangen.

Ein stärker auf der emotionalen Seite wirkender Ansatz bezieht sich auf Beschwerden und Störungen, die entweder wie bei vielen neurodegenerativen Erkrankungen - als unmittelbar neurogene Folge der cerebralen Veränderung oder als Reaktion auf die mit diesem «kritischen Lebensereignis» einhergehenden Einschnitte in das bisherige Leben entstehen (Anpassungsstörungen). Diese Indikation kann im Prinzip bei allen Erkrankungen des ZNS wie beispielsweise der Multiplen Sklerose, dem Schädel-Hirn-Trauma, cerebrovaskulären Ereignissen (Schlaganfällen, Hirnblutungen) sowie den Basalganglien- und neurodegenerativen Erkrankungen angezeigt sein.

\section{Die Umkehr des gelernten Nichtgebrauchs (learned non-use) als Beispiel der Restitution des somatischen Substrats}

Bei schlaganfallbedingten Paresen wird durch gezielte, lernpsychologisch begründete Trainingsmaßnahmen direkt auf die pathologisch veränderte neuronale Repräsentation einer geschädigten Extremität Einfluss genommen. Kommt es innerhalb einer cerebralen Hemisphäre zu einer regional begrenzten Mangelversorgung mit Sauerstoff, also einem «Insult» (stroke), führt dieses häufig zur Lähmung der kontralateralen Extremität. Taub [1976; 1980] zeigte jedoch, dass ein langfristiger Ausfall der Motorik keine alleinige Folge der neurogenen Schädigung, sondern darüber hinaus das Ergebnis eines Lernprozesses ist. So konnte bei nichthumanen Primaten nachgewiesen werden, dass sich die nach experimenteller Läsion einer Extremität einstellende Lähmung sehr viel schneller zurückbildet, wenn die gesunde Extremität noch während der Narkose mechanisch immobilisiert wurde. Bereits nach 3 Monaten waren die Funktionen des deafferentierten Arms dauerhaft restituiert [Taub, 2012].

Die Erklärung hierfür liegt im gelernten Nichtgebrauch: ist eine Extremität in ihrer Funktion eingeschränkt, ist jede Handlung nur mühsam zu vollziehen und führt oft nicht zum gewünschten Erfolg. Um diese «Bestrafung» des Handlungsversuchs zu umgehen, versucht ein Individuum automatisch die Kompensation mit der gesunden Extremität. Auf diese Weise werden die fehlerhaften Handlungsversuche der geschädigten Extremität umgangen und die Kompensationshandlung damit negativ verstärkt. Als Folge wird sehr schnell gelernt, die geschädigte Extremität nicht und die nichtgeschädigte Extremität kompensatorisch zu nutzen. Die Konsequenz eines langfristigen gelernten Nichtgebrauchs ist wiederum die allmähliche Rückbildung der cerebralen Repräsentation des geschädigten Organs. Gleichzeitig weiten sich die repräsentativen Felder der kompensatorisch eingesetzten Extremität entsprechend aus. Im Ergebnis kommt es zu einer langfristigen Verfestigung der Lähmung - als Folge eines Lernprozesses und nicht als unausweichliche Konsequenz einer neuronalen Schädigung; der Körper hat quasi «vergessen», wie das geschädigte Organ in Funktion gesetzt werden kann.

Bereits im Jahre 1904 wurde das Ausmaß einer schlaganfallbedingten Parese vom französischen Neurologen und Charcot-Schüler Henry Meige zumindest teilweise als Folge eines Verlustes des «motorischen Gedächtnisses» aufgefasst. Durch sorgfältige Krankenbeobachtungen gelang es ihm, die Abhängigkeit der Parese auf den postläsionellen Einsatz kognitiver und behavioraler Funktionen zurückzuführen. Meige sprach in diesem Zusammenhang von einer «motorischen Amnesie» [Meige, 1905]. In der englischen Übersetzung heißt es: «Numerous movements that were impossible in the initial stages of the illness subsequently became possible but are not carried out. The hemiplegic does not know them. He has forgotten them» [André et al., 2004]. Im Einzelnen sei die Parese «distinkt von läsioneller Paralyse», die Folge der Bewegungseinschränkung, an einen Lernprozess gekoppelt, mit einer Gedächtnisstörung einhergehend, reversibel und durch motorische Reedukation mit massierter und wiederholter Praxis behandelbar. Letzteres wurde im sogenannten Taubschen Training, dem «Constraint-Induced Movement Training» (CIMT), aufgenommen [Bauder et al., 2001]. Danach muss bei Patienten, bei denen in der geschädigten Extremität noch eine Restbeweglichkeit feststellbar ist, an 12 Tagen über jeweils $90 \%$ der Wachzeit die gesunde Extremität mit einer Schiene immobilisiet werden. Gleichzeitig werden sie angehalten, an den Wochentagen täglich 7 (mindestens 3) h intensiv mit dem paretischen Körperteil nach dem Prinzip der Ver- 
haltensformung (shaping) zu trainieren und schrittweise verschiedene Alltagsfunktionen einzuüben [Liepert et al., 2000; Miltner et al., 1999].

Dieses Training erzielt auch noch mehrere Jahre nach dem Apoplex Erfolge, allerdings führt ein früherer Beginn zu besseren Ergebnissen in der Feinmotorik [Lang et al., 2013]. In signifikantem, aber geringerem Ausmaß lässt sich das CIMT auch bei der unteren Extremität [Taub, 2012], bei motorischer Aphasie [Sickert et al., 2014], Balance- und Gangstörungen [Fuzaro et al., 2012] sowie der Cerebralparese des Kindesalters [Sterling et al., 2013] einsetzen. Taub selbst schätzt, dass bei der oberen Extremität 50\% aller Schlaganfallpatienten für eine CIMT infrage kommen und eine funktionelle Verbesserung um etwa 50\% erwarten können [Taub, 2012].

Bei einer entsprechenden Behandlung schlaganfallbedingter Wortproduktionsstörungen wird ebenfalls nach dem Prinzip der Verhaltensformung vorgegangen. So wird hier nur auf kommunikativ zielführende Lautbildungen positiv reagiert. Damit wird verhindert, dass die Umwelt allmählich die zunächst wenig verständlichen Äußerungen des Schlaganfallpatienten zu deuten lernt, sich somit darauf einstellt und dem Patienten auf diese Weise die Möglichkeit nimmt, seine Sprechfunktion intrinsisch und durch die positiven Reaktionen der sozialen Umwelt auch extrinsisch motiviert zu verbessern.

Nach heutigem Stand ist ein CIMT-Ansatz aber auf jeden Fall durch ein intensives Heimtraining (transfer package) zu ergänzen. Damit sind systematische und tägliche häusliche Verhaltenstrainings gemeint, die mit ansteigendem Schwierigkeitsgrad (shaping) auf «Verträgen» beruhen, die von Symptomtagebüchern, genauen Feststellungen der motorischen Funktionsfähigkeit und Feedback-Einheiten begleitet und über Telefoninterviews kontrolliert werden.

Meige praktizierte bereits einen dem heutigen «massierten Training» (Forced-Use Training; FuT) ähnlichen Ansatz. So hielt er seine Patienten zu regelmäßigem Training an: «We can teach them (...) using exercises adapted to defined goals and repeating these sufficiently, we manage to create these habits of motor associations (...), positive repercussions on both the motor apparatus (...) and the nerve centres and conductors; the organ benefits from exercising the function; finally the patient's moral is improved» [Meige, 1905; André et al., 2004].

Trotz der seit vielen Jahren nachgewiesenen guten Wirksamkeit des Taubschen Trainings und des seit über 100 Jahren verstandenen Wirkprinzips werden derartige verhaltensmedizinische Interventionen außerhalb von wenigen Universitätseinrichtungen praktisch nicht eingesetzt. Ein Grund hierfür liegt sicherlich in der sehr aversiven «Constraint»-Komponente, also der Verhaltensunterbindung der gesunden Extremität beim skizzierten CIMT. Allerdings hat die Forschung inzwischen gezeigt, dass der Verhaltensunterbindung eine geringere Bedeutung zukommt als dem gezielten Training der geschädigten Extremität. Hierzu gibt es im Vergleich zu den früheren, die Therapietreue der Patienten stark belastenden Restriktionsmethoden modifizierte Ansätze.
So kann auf die Constraint-Komponente dann verzichtet werden, wenn die situativen Kontingenzen so gesetzt werden, dass die betroffene Extremität genutzt werden muss. Dabei sind negative Verstärkungen durch den kompensatorischen Gebrauch der weniger betroffenen Extremität ebenso auszuschließen wie Bestrafungen von Handlungsversuchen, die mit dem paretischen Glied erfolgen. Der so erzwungene Gebrauch kann durch eine im Vergleich zur mechanischen Immobilisation weniger aversive Inhibition unterstützt werden, wenn die Beweglichkeit und Sensitivität der weniger betroffenen Hand z.B. mit einem Handschuh eingeschränkt wird.

Ein neuerer Ansatz beruht auf der Verwendung einer anästhesierenden Salbe, die an der betroffenen Extremität aufgetragen wird. Eine derartige temporäre funktionelle Deafferentierung (TFD) führt in Kombination mit einer intensiven Shaping-Prozedur in den contralateralen somatosensorischen Feldern offenbar zu einer erhöhten Exitabilität. Weiterhin erfolgt eine Reorganisation der rezeptiven kortikalen Areale, was $\mathrm{zu}$ einer verminderten interhemisphärischen Inhibition und damit letztlich zu einer verbesserten motorischen Funktion führt [Sens et al., 2012].

\section{Das Brain-Computer-Interface als Methode der Kompensation}

Eine besondere Form, Teilhabe zu ermöglichen, lässt sich beispielhaft bei Patienten mit Amyotropher Lateralsklerose (ALS) realisieren (Tab. 1). In den fortgeschrittenen Stadien weist diese Erkrankung eine nahezu komplette Lähmung der gesamten Willkürmotorik auf. Die kognitive Leistungsfähigkeit bleibt - zumindest bei der Mehrzahl der Patienten, bei denen die ALS nicht zusätzlich mit einer frontotemporalen Demenz assoziiert ist - bis auf leichte Beeinträchtigungen der Exekutivfunktionen jedoch weitgehend intakt [Röttig et al., 2006]. Wegen der in diesem Stadium nicht mehr gegebenen Möglichkeit der verbalen oder nonverbalen Kommunikation werden diese Patienten immer wieder als Beispiel für die moralische Berechtigung eines «Assistierten Suizides» angeführt. Seit es den Patienten mithilfe des skizzierten BCI möglich ist, über den eigenen emotionalen Status Auskunft $\mathrm{zu}$ geben, muss diese Ansicht jedoch grundlegend revidiert werden. So ist in besonders eindrücklicher Weise nicht nur der lediglich schwache Zusammenhang zwischen dem Grad der funktionellen Einschränkung und dem Abbau des Wohlbefindens, sondern vor allem die Abhängigkeit des psychischen Zustands von situativen Umständen deutlich geworden. Neben neueren Arbeiten, in denen bis in die finalen Stadien der ALS hinein eine immer differenziertere Konditionierbarkeit elektrischer Hirnpotenziale nachgewiesen wurde, finden sich heute vor allem Befunde zur emotionalen Situation der Erkrankten [Birbaumer et al., 2012; DeMassari et al., 2013; Münßinger et al., 2010; Halder et al., 2010; 2013; Silvoni et al., 2013]. 
Danach weisen schwer beeinträchtigte ALS-Patienten dann eine gute Lebensqualität auf, wenn das soziale Netzwerk intakt ist und als fürsorglich erlebt wird. Verwandte und Pflegekräfte neigen dazu, die Depression der Patienten zu überund die Lebensqualität zu unterschätzen. Auch der Wunsch nach einem vorgezogenen Tod wird von den Angehörigen systematisch überschätzt. Selbst die dauerhafte assistierte Beatmung geht nicht systematisch mit Selbstaufgabe, sondern im Gegenteil, häufig eher mit dem Erhalt des Lebensmutes einher. Zusammengefasst sind psychische Störungen selten, und subsyndromale Ausprägungen psychischer Beschwerden weisen eine nur geringe bis mäßige Korrelation mit dem motorischen Funktionsverlust auf [Matuz et al., 2010]. Dagegen weist die Studienlage konsistent auf erhebliche Depressionsund andere Belastungswerte bei den Angehörigen hin, die zur systematischen Überschätzung der Belastung von Patienten führen.

Aufgrund dieser Befunde hat sich heute die Ansicht durchgesetzt, dass auch das zeitlich begrenzte Leben mit einer ALS von den Patienten selbst als lebenswert empfunden wird und dass psychologische Hilfen für die Patienten, insbesondere aber auch für die Angehörigen, zu einer signifikanten Besserung der Lebensqualität beitragen. Es ist also eine Frage des psychosozialen Umfelds, der Möglichkeit aktiver Kommunikation (gegebenenfalls mithilfe eines BCI) und der physischen Umfeldgestaltung, weniger der Erkrankungsschwere und ob ein Leben auch in derartigen schweren und finalen Phasen noch als sinnvoll empfunden wird [Lillo et al., 2012; Pagnini, 2013; Lulé et al., 2009; 2013; Rabkin et al., 2005; 2006; 2009; Gauthier et al., 2007; Trai et al., 2003; Kübler et al., 2005; Rousseau et al., 2013; Chiò et al., 2005].

Eine derartige Dissoziation von Funktionsbeeinträchtigung und mangelnder sozialer Teilhabe zeigt eindrücklich, dass auch von schwersten Beeinträchtigungen nicht automatisch auf eine Verminderung der Lebensqualität geschlossen werden darf. Entsprechend führt jedoch auch die Verbesserung der Motorik nicht zwangsläufig zu einer anhaltenden Verbesserung der Emotionalität. Dieses kann heute beispielhaft bei den Basalganglienerkrankungen (Parkinson, Dystonie, Tremor) beobachtet werden, bei denen eine Tiefe Hirnstimulation (THS) durchgeführt wurde. Trotz der zumeist erheblichen Verbesserungen der motorischen Funktionen finden sich im Langzeitverlauf bei etwa einem Drittel der untersuchten Parkinson-Patienten und ihren Angehörigen erhebliche emotionale und zwischenmenschliche Konfliktkonstellationen. Diese Auswirkungen waren nicht mit dem Operationserfolg korreliert, sondern resultierten zu einem großen Teil aus dem kognitiven und psychischen Anpassungsniveau des präoperativen Zustands. Auch die Erwartungen, die subjektive Lebensbilanz und die partnerschaftliche Situation determinierten entscheidend die bewerteten Langzeiteffekte der THS - die nach objektiver Messung in der Regel hervorragend ausfielen [Maier et al., 2013; Schüpbach et al., 2006; Agid et al., 2006].
BCIs werden neuerdings auch in der Rehabilitation von Schlaganfallpatienten eingesetzt [Teo und Chew, 2014]. Dabei werden die bei einer Vorstellung der motorischen Handlung entstehenden Veränderungen der sensomotorischen Oszillationen erfasst und zur Steuerung des BCIs genutzt. Wie auch im Einsatz bei ALS- oder Locked-in-Patienten gilt jedoch, dass nicht alle Patienten von dieser Hilfe profitieren. Von verhaltensmedizinischer Bedeutung ist jedoch die Tatsache, dass dies - entgegen früherer Lehrmeinungen - überhaupt möglich ist. Mit künftigen Forschungen wird herauszufinden sein, bei welchen Patienten welche Form der Kompensation und Restitution mit welcher Aussicht auf Erfolg eingesetzt werden kann.

\section{Selbstregulation durch die Steigerung situations- spezifischer Symptomkontrolle}

Die Möglichkeiten einer situationsspezifischen Symptomkontrolle lassen sich beispielhaft bei den Anfallserkrankungen zeigen. Den EEG-Feedback-Verfahren lag ursprünglich die Beobachtung zugrunde, dass die über dem sensomotorischen Cortex ableitbare 12-15 Hz starke rhythmische Aktivität (sensorimotorische Rhythmen; SMR) den Schlafspindeln bei ruhigem Schlaf ähnelt. Erste Versuche einer operanten Konditionierung dieser Aktivität führten zu dem Zufallsbefund eines anhaltenden antikonvulsiven Effekts [Sterman, 2010; Strehl, 1998]. Die klinisch bedeutsame Reduktion der Anzahl und Schwere epileptischer Anfälle konnte in zahlreichen Folgestudien bestätigt werden [Tan et al., 2009], sodass die operante Kontrolle sensorimotorischer Rhythmen heute als ein etablierter Weg der gelernten Anfallskontrolle gelten kann. Ein weiterer EEG-Feedback-Ansatz beruht auf der an der Schädeloberfläche erfassten Positivierung sogenannter Langsamer Kortikaler Potenziale (Slow Cortical Potentials; SCPs), die mit einer vermehrten Anfallskontrolle einhergeht [Strehl et al., 2006]. Dabei scheint das Ausmaß einer anhaltenden Negativierung der SCPs mit einem schlechteren Übungserfolg assoziiert zu sein.

Ohne technische Hilfen ist eine situationsspezifische Verhaltensregulation bei allen neurologischen Symptomen anwendbar, die sich durch situations- oder emotionsspezifische Exazerbationen auszeichnen (z.B. bei epileptischen Anfällen). Diese verteilen sich nicht zufällig über den Tag hinweg, sondern entstehen nach identifizierbaren Auslösereizen. Einer neueren Erhebung zufolge [Balamurugan et al., 2013] sind dies vor allem Unregelmäßigkeiten in der Medikamenteneinnahme (40,9\%), emotionale Belastungen (31,3\%), Schlafmangel (19,7\%), Müdigkeit (15,3\%), ausgelassene Mahlzeiten (9,1\%), Fieber $(6,4 \%)$ und Rauchen (6,4\%); gelegentlich wird auch noch die Menstruation genannt. Das Erkennen derartiger Auslöser lässt sich schulen, und über den kontingenten Einsatz der bereits genannten Verhaltensalternativen kann ein beginnender Anfall blockiert werden [Andrews et al., 2000; 
Ferwick, 1994; Spector et al., 1999; Schmidt-Schönbein, 1998; Strehl, 1998]. Gegebenenfalls ist eine Unterstützung der psychophysiologischen Selbstregulation durch elektrodermales Biofeedback hilfreich [Nagai und Trimble, 2014]. Auch kann der genannte EEG-Feedback-Einsatz mit der Schulung situativer und internaler Auslöser und dem Einsatz kompensatorischer Verhaltensmanöver kombiniert werden.

Ein weiterer Ansatzpunkt spezifischer Selbstkontrollmaßnahmen besteht in den situationsspezifischen Symptom-Exazerbationen, wie sie bei Patienten mit Morbus Parkinson und anderen Basalganglienerkrankungen zum klinischen Alltag gehören. Hier geht es darum, bestimme Symptome wie z.B. den Parkinson-Tremor oder situativ exazerbierende Symptome bei den Dystonien unter Verhaltenskontrolle zu bringen. Dies geschieht ähnlich wie bei den Epilepsien durch eine genaue Situationsanalyse mit der Identifikation auslösender Stimuli und der sie begleitenden dysfunktionalen Kognitionen. Im Folgeschritt werden die diesen Auslösern üblicherweise folgenden Vermeidungsverhaltensweisen unterbunden und durch gezielte Expositionsmaßnahmen ersetzt. Dabei wird das «Vermeiden des Vermeidens» sowohl mit Maßnahmen zur sozialen Kompetenz als auch mit Strategien zur Blockade überschießender motorischer Symptome kombiniert. Dadurch wird der sich aufschaukelnde Prozess von situationsspezifischer Exazerbation, Angst und Unruhe, weiterer symptomatischer Exazerbation und Rückzugsverhalten mit dem daraus resultierenden weiteren Anstieg sozialer Unsicherheit, Rückzug und emotionaler Belastung effektiv unterbunden [Leplow, 2007; 2011a].

\section{Selbstregulation durch die Beeinflussung von Emotionalität und Krankheitsverhalten}

In der neueren Literatur finden sich vor allem Arbeiten, die auf die Lebenssituation der Betroffenen zielen. So werden mithilfe kognitiv-verhaltenstherapeutischer Methoden erkrankungsassoziierte Depressionen sowie Ängste und Beeinträchtigungen der Lebensqualität behandelt. Hier wird mit den bewährten Vorgehensweisen der Kognitiven Verhaltenstherapie (Cognitive Behavioral Therapy; CBT) relativ unabhängig von der konkreten Erkrankung der verminderten Teilhabe am sozialen Leben entgegengewirkt (Tab. 1, rechter Teil).

Das zeigt sich insbesondere bei epileptischen Erkrankungen. Wurden in der Vergangenheit fast ausschließlich anfallszentrierte Maßnahmen praktiziert, finden sich derzeit vorwiegend kognitiv-behaviorale Interventionen. Diese wirken zwar mit großem Erfolg auf die emotionalen Begleiterscheinungen der Erkrankung, jedoch haben sie auf die Charakteristika der epileptischen Anfälle weniger Einfluss [Mula, 2014; CrailMeléndez, et al., 2012; Macrodimitris et al., 2011; Goldstein et al., 2003; Ramaratnam et al., 2008; Gandy et al., 2013; Tang et al., 2014; May und Pfäfflin, 2002; Ried et al., 2001; SchmidtSchönbein, 1998].
Auch bei der Multiplen Sklerose haben sich kognitiv-behaviorale Therapien insbesondere mit Fokus auf depressive Störungen und die erkrankungsspezifische Müdigkeit (Fatigue) bewährt. Die Wirksamkeit zeigte sich hier auch im Vergleich $\mathrm{zu}$ emotionsfokussierten und supportiven Interventionen sowie Entspannungsübungen, da die CBT vor allem über die Reduktion des Vermeidungsverhaltens sowie die Veränderung dysfunktionaler Einstellungen zur Fatigue wirkt [Hind et al., 2014; Moss-Morris et al., 2013; Knoop et al., 2012]. Für die Versorgungswirklichkeit ist es von Bedeutung, dass sich der Einsatz der CBT auch über Telefon [Cosio et al., 2011] oder das Internet [Moss-Morris et al., 2012] bewährt hat. Allerdings müssen subgruppenspezifische Effekte noch auf spezifische Wirkfaktoren im Langzeitverlauf hin untersucht werden.

Entsprechend sind die verhaltensmedizinischen Ansätze bei den Basalganglienerkrankungen, insbesondere bei Morbus Parkinson zu bewerten. Ausgehend von einer besseren Differenzialdiagnostik der unterschiedlichen psychischen Syndrome (unter anderem subsyndromale (subthreshold) Angst- und Depressionssyndrome, sekundäre vs. primäre Syndrome, Apathie, Anpassungsstörungen, somatopsychische Wechselwirkung im Sinne der ICD-Diagnose F54, etc.) stehen hier vor allem die krankheitsbedingten Belastungen der Patienten und ihrer Angehörigen sowie das damit einhergehende Vermeidungsverhalten im Vordergrund. Diese Interventionen werden gleichermaßen bei den psychischen Syndromen eingesetzt, die als «reaktiv» angesehen werden (Anpassungsstörungen als Folge des Diagnoseschocks oder Phasen der Verschlechterung), als auch bei den Angst- und affektiven Störungen, die als unmittelbare Folge einer Neurodegeneration auftreten können (Störung aufgrund eines medizinischen Krankheitsfaktors). Nach inzwischen knapp 30 Beobachtungsstudien (davon 7 kontrollierte klinische Studien) kann die Wirksamkeit kognitiv-behavioraler Maßnahmen als belegt gelten [Leplow, 2011a]. Neuerdings werden darüber hinaus auch die sogenannten Impulskontrollstörungen, die als Nebenwirkung dopaminerger Medikation entstehen können und bis vor Kurzem für psychologische Interventionen als unzugänglich galten, mit kognitiv-behavioralen Methoden behandelt [Okai et al., 2013]. Eine spezielle Anpassung der CBT auf die spezifischen Besonderheiten der Dystonien steht allerdings noch aus.

Auch bei den Demenzen kommen zunehmend psychologisch fundierte Programme und Vorgehensweisen zum Einsatz [Orgeta et al., 2014]. Diese nutzen oft Kompensationsstrategien, bei denen beispielsweise versucht wird, die Auswirkungen der sehr früh einsetzenden Schädigung der Hippocampus-Formation auf komplexe Lern- und Orientierungsleistungen durch die Etablierung einfacher Reiz-Reaktionssequenzen abzumildern. Andere Methoden zielen auf Veränderungen situativer Kontextvariablen, wodurch demenzbedingte Verhaltensstörungen unter Stimuluskontrolle gebracht werden können. Ansonsten liegt der Fokus auf der Bildung Gedächtnis entlastender Strategien, der Nutzung sehr alter und häufig reaktivierter Erinnerungen und dem Aufbau krankheits- 
angepasster Alltagsroutinen. In dem ersten vorliegenden Therapiemanual [Werheid und Thöne-Otto, 2006] wird vor allem Wert gelegt auf die Etablierung Gedächtnis schonender Routinen durch Gewohnheitsbildungen, die Stärkung des «Selbsterhalts» durch «persönliche Erinnerungsschätze» und damit die Nutzung der «Kraftquelle Lebensgeschichte». Ein Cochrane-Review über randomisierte klinische Studien [Woods et al., 2012] ergab, dass sich eine psychologische Intervention günstig auf die Depression bei demenzkranken Patienten auswirkt. Darüber hinaus zeigte sich, dass kognitive Stimulation bei frühen Formen der Demenz die Effekte der Pharmakotherapie weiter verbessern kann.

\section{Der Sonderfall der "psychogenen" Störungen in der Neurologie}

In der neurologischen Literatur trifft man häufig auf den Terminus der «psychogenen» Störung, der besonders bei Anfalls- oder Bewegungsstörungen, aber auch bei Erkrankungen sensorischer Systeme vergeben wird, wenn die präsentierte Symptomatik nicht den bekannten neurologischen Kriterien der infrage stehenden Erkrankung entspricht. Sind darüber hinaus eine Abhängigkeit der Symptommanifestation oder Phasen der Symptom-Exazerbation von emotionalen oder lebensgeschichtlichen Ereignissen feststellbar, wird üblicherweise die Diagnose einer psychogenen Störung vergeben. Eine derartige Festsetzung wird von vielen Patienten und ihren Organisationen als stigmatisierend empfunden und entspricht nicht den klassifikatorischen Möglichkeiten psychischer Störungen. Bereits im DSM-IV als auch im noch gültigen ICD-10 wären hierfür die «Konversionsstörung» (DSM-IV) oder «Dissoziative Bewegungsstörung» (ICD-10), gegebenenfalls auch eine «Undifferenzierte Somatoforme Störung» oder eine «Somatopsychische Wechselwirkung» gemäß der Kategorie F54 des ICD-10 (bzw. der Kategorie 316 im DSM-IV) zu vergeben.

Schwerwiegender ist die konzeptuelle Unhaltbarkeit des Begriffs einer psychogenen Störung. Dieser enthält im Wortsinn eine wie auch immer geartete psychogene Ätiologie, welche die überholte Körper-Psyche-Dichotomie wieder aufleben lässt, das Gehirn als Grundlage unseres Verhaltens und Erlebens ignoriert und nicht unser heutiges multifaktorielles Verständnis der Entstehung von Krankheiten berücksichtigt. Auch widerspricht eine derartige Diagnose dem rein deskriptiven Charakter derzeitiger Diagnosesysteme. Ausgenommen sind hierbei wenige Störungen wie z.B. die Posttraumatische Belastungsstörung (PTBS), substanzbezogene Störungen, Anpassungsstörungen, Störungen aufgrund eines medizinischen Krankheitsfaktors, etc.

Dieser Umstand wurde in dem Ende 2013 erschienenen DSM-5 berücksichtigt. Hier werden die bislang als unklar oder psychogen angesehenen Störungen in der Kategorie der «Somatic Symptom and Related Disorders» gefasst. Im Ge- gensatz zu der im ICD-10 unter F54 kodierten Störung der «Psychologischen Faktoren oder Verhaltensfaktoren bei andernorts klassifizierten Krankheiten», in der «Verhaltenseinflüsse (...), die wahrscheinlich eine wesentliche Rolle in der Manifestation körperlicher Krankheiten spielen» von diagnostischer Bedeutung sind, spielt die Verursachungsannahme im DSM-5 ausdrücklich keine Rolle mehr. Der Fokus liegt hier auf dem somatischen Syndrom. Die Abwesenheit einer medizinischen Erklärung ist für die Klassifikation ausdrücklich ohne Bedeutung. Es handelt sich hiermit also um eine positive Diagnose und nicht mehr um eine Ausschlussdiagnose. Im Zentrum der Diagnostik steht fortan der affektive, kognitive und behaviorale Umgang mit einer Erkrankung. Äußert sich dieser dysfunktional, erzeugt er weiteren Leidensdruck, zieht zusätzliche (psychosoziale) Beeinträchtigung nach sich, und somit kann entweder das Störungsbild der «Conversion Disorder / Functional Neurologic Symptom Disorder» oder das der «Psychological Factors Affecting Other Medical Conditions» diagnostiziert werden.

Im ersteren Fall werden insbesondere Anfallserkrankungen oder Bewegungsstörungen (Basalganglienerkrankungen) genannt, deren Symptomatik nicht den klassischen Beschreibungen der scheinbar zugrunde liegenden somatischen Erkrankungen entspricht. Allerdings gilt hier die Vorstellung einer - multifaktoriell bedingten - Funktionsstörung des ZNS ohne Referenz auf eine bestimmte Ätiologie. Solche Funktionsstörungen können sich durchaus im Erscheinungsbild einer der bekannten ZNS-Erkrankungen präsentieren. Ob sie jedoch Varianten dieser Krankheiten darstellen oder gänzlich anders $\mathrm{zu}$ verstehen sind, ist diagnostisch unerheblich und Gegenstand weiterer Forschung.

Noch klarer wird diese veränderte Vorstellung der ehemals als psychogen genannten Störungen in der Diagnose, die im Englischen «Psychological Factors Affecting Medical Conditions» genannt wird. Auch hier wird explizit vom Vorhandensein eines somatischen Symptoms ausgegangen. Diese Diagnose wird vergeben, wenn Verhaltensfaktoren bei der Manifestation oder dem Verlauf, bei der Inanspruchnahme der medizinischen Behandlung oder dem Gesundheitsverhalten eine signifikante Rolle spielen oder diese Faktoren auf andere Art und Weise auf die somatische Pathologie einwirken (z.B. durch den Anstieg des Parkinson-Tremors nach Aufregung und Scham) [Leplow 2008a,b; 2011b; Leplow und Wiemann, 2010; Leplow, et al., 2013].

Damit gibt es heute zumindest über das DSM-5 gute diagnostische Möglichkeiten, «das Psychische» eines somatischen Krankheitsbildes korrekt zu fassen, ohne dass auf ein veraltetes Psychogenese-Konzept zurückgegriffen werden muss.

\section{Fazit}

Zusammengefasst ergeben sich für verhaltensmedizinische Interventionen zahlreiche Indikationen, die sowohl direkt auf 
das somatische Substrat als auch auf die Auswirkungen der somatischen Funktionseinschränkung zielen. Bereits jetzt liegen vielfältige Möglichkeiten für den Einsatz überprüfter verhaltensmedizinischer Behandlungen vor, die ständig weiterentwickelt werden.

Gerade vor dem Hintergrund der demografischen Entwicklung wird ohne die gezielte Förderung der Selbstregulation physiologischer und behavioraler Systeme keine adäquate Krankenbehandlung mehr möglich sein. Die Aufgabe der im Bereich der Verhaltensmedizin Tätigen wird es daher sein, die vorhandenen Möglichkeiten auszubauen, Indikationen und Kontraindikationen zu benennen und das Wissen über diese Möglichkeiten auf allen Ebenen des Gesundheitssystems zu verbreiten. Darüber hinaus stellt sich für die Zukunft als besondere Herausforderung, die verhaltensmedizinischen Möglichkeiten auf die unterschiedlichen Rahmenbedingungen der Versorgungswirklichkeit hin anzupassen.

\section{Dank}

Ich danke Frau Anna Eggebrecht, BSc in Psychologie, für die redaktionelle Bearbeitung des Manuskripts.

\section{Disclosure Statement}

Der Autor erklärt hiermit, dass keine Interessenskonflikte in Bezug auf das Manuskript bestehen.

\section{Literatur}

Agid Y, Schüpbach M, Gargiulo M, Mallet L, Houeto JL, Behar C, Maltête D, Mesnage V, Welter ML: Neurosurgery in Parkinson's disease: the doctor is happy, the patient less so? J Neural Transm Suppl 2006;70:409-414.

André J-M, Didier J-P, Paysant J: «Functional motor amnesia» in stroke (1904) and «learned non-use phenomenon» (1966). J Rehabil Med 2004;36:138-140.

- Andrews JD, Reiter JM, Schonfeld W, Kastl A, Denning P: A neurobehavioral treatment for unilateral complex partial seizure disorders: a comparison of right- and left-hemisphere patients. Seizure 2000; 9:189-197.

Balamurugan E, Aggarwal M, Lamba A, Dang N, Tripathi M: Perceived trigger factors of seizures in persons with epilepsy. Seizure 2013;22:743-747.

-Bauder H, Taub E, Miltner W: Behandlung motorischer Störungen nach Schlaganfall: die Taubsche Bewegegungsinduktionstherapie. Göttingen, Hog refe, 2001.

Birbaumer N, Piccione F, Silvioni S, Wildgruber M: Ideomotor silence: the case of complete paralyses and brain-computer interfaces (BCI). Psychol Res 2012;76:183-191.

Chiò A, Gauthier A, Calvo A, Ghiglione P, Mutani R: Caregiver burden and patients' perception of being a burden in ALS. Neurology 2005;64:1780-1782.

Cosio D, Jin L, Siddique J, Mohr DC: The effect of telephone-administered cognitive-behavioral therapy on quality of life among patients with multiple sclerosis. Ann Behav Med 2011;41:227-234.

Crail-Meléndez D, Herrera-Melo A, Martinez-Juárez IE, Ramirez-Bermúdez J: Cognitive-behavioral therapy for depression in patients with temporal lobe epilepsy: a pilot study. Epilepsy Behav 2012; 23:52-56.

DeMassari D, Matuz T, Furdea A, Ruf CA, Halder S, Birbaumer N: Brain-computer interface and semantic classical conditioning of communication in paralysis. Biol Psychol 2013;92:267-274.

Ferwick P: The behavioral treatment of epilepsy generation and inhibition of seizures. Neurol Clin 1994;12:175-202.

- Fuzaro AC, Guerreiro CT, Galetti FC, Juca RB, Araujo JE: Modified constraint-induced movement therapy and modified forced-use therapy for stroke patients are both effective to promote balance and gait improvements. Rev Bras Fisioter 2012;16:157-165.
Gandy M, Sharpe L, Perry KN: Cognitive behavior therapy for depression in people with epilepsy: a systematic review. Epilepsia 2013;54:1725-1734.

Gauthier A, Vignola A, Calvo A, Cavallo E, Moglia C, Sellitti L, Mutani R, Chiò A: A longitudinal study on quality of life and depression in ALS patientcaregiver couples. Neurology 2007;68:923-926.

Goldstein LH, McAlpine M, Deale A, Toone BK, Mellers JD: Cognitive behaviour therapy with adults with intractable epilepsy and psychiatric comorbidity: preliminary observations on changes in psychological state and seizure frequency. Behav Res Ther 2003;41:447-460.

Halder S, Hammer EM, Kleih SC, Bogdan M, Rosenstiehl W, Birbaumer N, Kübler A: Prediction of auditory and visual p300 brain-computer interface aptitude. PLoS One 2013;8:e53513.

Halder S, Rea M, Andreoni R, Nijboer F, Hammer EM, Kleih SC, Birbaumer N, Kübler A: An auditory oddball brain-computer interface for binary choices. Clin Neurophysiol 2010;121:516-532.

Hind J, Cotter J, Thake A, Bradburn M, Cooper C, Isaac C, House A: Cognitive behavioural therapy for the treatment of depression in people with multiple sclerosis: a systematic review and meta-analysis. BMC Psychiatry 2014;14:5.

Knoop H, van Kessel K, Moss-Morris R: Which cognitions and behaviours mediate the positive effect of cognitive behavioural therapy on fatigue in patients with multiple sclerosis? Psychol Med 2012; 42:205-213.

Kübler A, Winter S, Ludolph SC, Hautzinger M, Birbaumer N: Severity of depressive symptoms and quality of life in patients with amyotrophic lateral sclerosis. Neurorehabil Neural Repair 2005;19:182193.

Lang KC, Thompson PA, Wolf SL: The EXCITE Trial: reacquiring upper-extremity task performance with early versus late delivery of constraint therapy. Neurorehabil Neural Repair 2013;27:654-663.

Leplow B: Parkinson. Fortschritte der Psychotherapie. Göttingen, Hogrefe, 2007.

Leplow B: Dystonie, Tic und Tremor; in Röhrle B, Caspar PF, Schlottke P (eds): Lehrbuch der Klinisch-Psychologischen Diagnostik. Stuttgart, Kohlhammer, 2008a, pp 843-866.

Leplow B: Parkinson und Dystonie: Verhaltensmedizin der Basalganglienerkrankungen; in v. Leupoldt A, Ritz T (eds): Lehrbuch der Verhaltensmedizin. Stuttgart, Kohlhammer, 2008b, pp 325-340.
Leplow B: Morbus Parkinson; in Hautzinger M (ed): Kognitive Verhaltenstherapie bei psychischen Störungen, ed 4. Weinheim, Beltz, 2011a.

Leplow B: Primäre Dystonien - zwischen Botulinum Toxin-Behandlung, Neuropsychologie und Tiefer Hirnstimulation. Z Neuropsychol 2011b;22:167-180.

Leplow B, Böttcher M, Schönfeld R: BotulinumToxin-Therapie bei Torticollis spasmodicus. Nervenarzt 2013;84:493-497.

Leplow B, Wiemann J: Zwischen Disability und Handicap. Zur Frage der Therapietreue von TorticollisPatienten bei der Therapie mit Botulinumtoxin. Nervenheilkunde 2010;28:519-525.

Liepert J, Bauder H, Wolfgang HR, Miltner WH, Taub E, Weiller C: Treatment-induced cortical reorganization after stroke in humans. Stroke 2000; 31:1210-1216.

Lillo P, Mioshi E, Hodges JR: Caregiver burden in amyotrophic lateral sclerosis is more dependent on patients' behavioral changes than physical disability: a comparative study. BMC Neurol 2012;12:156.

Lulé D, Ehlich B, Lang D, Sorg S, Heimrath J, Kübler A, Birbaumer N, Ludolph AC: Quality of life in fatal disease: the flawed judgement of the social environment. J Neurol 2013;260:2836-2843.

Lulé D, Zickler C, Häcker S, Bruno MA, Demertzi A Pellas F, Laureys S, Kübler A: Life can be worth living in locked-in syndrome. Prog Brain Res 2009; 177:339-351.

Macrodimitris S, Wershler J, Hatfield M, Hamilton K, Backs-Dermott B, Mothersill K, Mothersill K, Baxter C, Wiebe S: Group cognitive-behavioral therapy for patients with epilepsy and comorbid depression and anxiety. Epilepsy Behav 2011;20:83-88.

Maier F, Lewis CJ, Horstkoetter N, Eggers C, Kalbe E, Maarouf M, Kuhn J, Zurowski M, Moro E, Woopen C, Timmerman L: Patients' expectations of deep brain stimulation, and subjective perceived outcome related to clinical measures in Parkinson's disease: a mixed-method approach. J Neurol Neurosurg Psychiatry 2013;84:1273-1781.

Matuz T, Birbaumer N, Hautzinger M, Kübler A: Coping with amyotrophic laterals sclerosis: an integrative view. J Neurol Neurosurg Psychiatry 2010;81:893-898.

May TW, Pfäfflin M: The efficacy of an educational treatment program for patients with epilepsy (MOSES): results of a controlled, randomized study. Epilepsia 2002;43:539-549. 
Meige, H: Les amnésies motrices fonctionnelles et le traitement de hémiplégiques. Rev Neurol (Paris) 1905;13:183-184.

-Miltner WH, Bauder H, Sommer M, Dettmers C, Taub E: Effects of constraint-induced movement therapy on patients with chronic motor deficits after stroke: a replication. Stroke 1999;30:586-593.

Moss-Morris R, Dennison L, Landau S, Yardley L, Silber E, Chalder T: A randomized controlled trial of cognitive behavioral therapy (CBT) for adjusting to multiple sclerosis (the saMS trial): does CBT work and for whom does it work? J Consult Clin Psychol 2013;81:251-262.

- Moss-Morris R, McCrone P, Yardley L, van Kessel K, Wills G, Dennison L: A pilot randomised controlled trial of an Internet-based cognitive behavioural therapy self-management programme (MS Invigor8) for multiple sclerosis fatigue. Behav Res Ther 2012;50:415-421.

Münßinger JI, Halder S, Kleih SC, Furdea A, Raco V, Hösle A, Kübler A: Brain painting: first evaluation of a new brain-computer interface application with ALS-patients and healthy volunteers. Front Neurosci 2010;22:182.

Mula M: Epilepsy-induced behavioral changes during the ictal phase. Epilepsy Behav 2014;30:14-16.

Nagai J, Trimble MR: Long-term effects of electrodermal biofeedback training on seizure control in patients with drug-resistant epilepsy: two case reports. Epilepsy Res 2014;108:149-152.

Dokai D, Askey-Jones S, Samuel M., O'Sullivan SS, Chaudhuri KR, Martin A, Mack J, Brown RG, David AS: Trial of CBT for impulse control behaviours affecting Parkinson patients and their caregivers. Neurology 2013;80:782-799.

Dorgeta V, Qazi A, Spector AE, Orrell M: Psychological treatments for depression and anxiety in dementia and mild cognitive impairment. Cochrane Database Syst Rev 2014;22:CD009125.

Pagnini F: Psychological wellbeing and quality of life in amyotrophic lateral sclerosis: a review. Int J Psychol 2013;48:194-205.

-Rabkin JG, Albert SM, DelBene ML, O'Sullivan I, Tider T, Rowland LP, Mitsumoto H: Prevalence of depressive disorders and change over time in latestage ALS. Neurology 2005;65:62-67.

Rabkin JG, Albert SM, Rowland LP, Mitsumoto H How common is depression among ALS caregivers? A longitudinal study. Amyotroph Lateral Scler 2009;10:448-455.
Rabkin JG, Albert SM, Tider T, DelBene ML, O'Sullivan I, Rowland LP, Mitsumoto H: Predictors and course of elective long-term mechanical ventilation: a prospective study of ALS patients. Amyotroph Lateral Scler 2006;7:86-95.

Ramaratnam S, Baker GH, Goldstein LH: Psychological treatments for epilepsy. Cochrane Database Syst Rev 2008;16:CD002029.

Ried S, Specht U, Thorbecke R, Goecke K, Wohlfarth R: MOSES: an educational program for patients with epilepsy and their relatives. Epilepsia 2001;42 (suppl 3):76-80.

Röttig D, Leplow B, Eger K, Ludolph AC, Graf M, Zierz S: Only subtle cognitive deficits in non-bulbar amyotrophic lateral sclerosis patients. J Neurol 2006;253:333-339.

Rousseau MC, Pietra S, Nadji M, Billette deVillemeur $\mathrm{T}$ : Evaluation of quality of life in complete lockedin-syndrome patients. J Palliat Med 2013;16:14551458.

Schmidt-Schönbein C: Improvement of seizure control by psychological methods in patients with intractable epilepsies. Seizure 1998;7:261-270.

-Schüpbach M, Gargiulo M, Welter ML, Mallet L, Béhar C, Houteo JL, Maltête D, Mesagne V, Agid, Y: Neurosurgery in Parkinson disease: a distressed mind in a repaired body? Neurology 2006;66:1811-1816.

Sens E, Teschner U, Meisser W, Preul C, Huonker R, Witte OW, Miltner WH, Weiss T: Effects of temporary functional deafferentation on the brain, sensation, and behavior of stroke patients. J Neurosci 2012;32:11773-11779.

Sickert A, Anders LC, Münte TF, Sailer M: Constraint-induced aphasia therapy following subacute stroke: a single-blind, randomised clinical trial of a modified therapy schedule. J Neurol Neurosurg Psychiatry 2014;85:51-55.

Silvoni S, Cavinato M, Volpato C, Ruf CA, Birbaumer N, Piccione F: Amyotrophic lateral sclerosis progression and stability of brain-computer interface communication. Amyothroph Lateral Scler Frontotemporal Degener 2013;14:390-396.

Spector S, Tranah A, Cull C, Goldstein LH: Reduction in seizure frequency following a short-term group intervention for adults with epilepsy. Seizure 1999; 8:297-303.
Sterling C, Taub E, Davis D, Rickards T, Gauthier LV, Griffin A, Uswatte G: Structural neuroplastic change after constraint-induced movement therapy in children with cerebral palsy. Pediatrics 2013;131: 1664-1669.

Sterman MB: Biofeedback in the treatment of epilepsy. Cleve Clin J Med 2010;3:60-67.

Strehl U: Epilepsie und Verhalten. Entwicklung und Prüfung eines psychophysiologischen Behandlungsprogramms zur Selbstkontrolle epileptischer Anfälle. Lengerich, Pabst, 1998.

Strehl U, Trevorrow T, Veit R, Hinterberger T, Kotchoubey B, Erb M, Birbaumer N: Deactivation of brain areas during self-regulation of slow cortical potentials in seizure patients. Appl Psychophysiol Biofeedback 2006;31:85-94.

Tan G, Thornby J, Hammond DC, Strehl U, Canady B, Arnemann K, Kaiser DA: Meta-analysis of EEG biofeedback in treating epilepsy. Clin EEG Neurosci 2009;40:173-179.

Tang V, Michaelis R, Kwan P: Psychobehavioral therapy for epilepsy. Epilepsy Behav 2014;32:147-155.

Taub E: Movement in nonhuman primates deprived of somatosensory feedback. Exerc Sport Sci Rev 1976;4:335-374.

Taub E: Somatosensory deafferentation research with monkeys: implications for rehabilitation medicine; in Ince LP (ed): Behavior Psychology in Rehabilitation Medicine: Clinical Applications. New York, Williams and Wilkins, 1980, pp 371-401.

Taub E: The behavior-analytic origins of constraintinduced movement therapy: an example of behavioral neurorehabilitation. Behav Anal 2012;35:155178.

Teo WP, Chew E: Is motor-imagery brain-computer interface feasible in stroke rehabilitation? PM R 2014;pii:S1034-1482(14)00021-5.

Trai M, Nelson MD, Van JN, Appel SH, Lai EC: A study comparing patients with amyotrophic lateral sclerosis and their caregivers on measures of quality of life, depression, and their attitudes toward treatment options. J Neurol Sci 2003;209:79-85.

Werheid K, Thöne-Otto A: Kognitives Training bei Alzheimer-Demenz. Der Nervenarzt 2006;77:549_ 557.

Woods B, Aguirre E, Spector AE, Orrell M: Cognitive stimulation to improve cognitive functioning in people with dementia. Cochrane Database Syst Rev 2012;15:CD005562. 